\title{
Existence of a regular solution for 1D Green-Naghdi equations with surface tension at a large time instant
}

\author{
Mohammad Haidar ${ }^{1 *}$, Toufic El Arwadi ${ }^{1}$ and Samer Israwi ${ }^{2}$
}

\section{"Correspondence:}

mohammadhaidar2288@gmail.com

${ }^{1}$ Department of Mathematics and

Computer Science, Faculty of

Science, Beirut Arab University, Beirut, Lebanon

Full list of author information is available at the end of the article

\begin{abstract}
In this paper the model 1D-GNo is considered, which concerns the 1D Green-Naghdi equations with non-flat bottom and under the influence of surface tension, to be widely used in coastal oceanography to describe the propagation of large-wave amplitudes. The purpose of this paper is to show that the solution of 1D-GN $\sigma$ can be made by the Picard iterative scheme, which proves that there is no loss of regularity of the solution relative to the initial condition.
\end{abstract}

Keywords: Water waves; Green-Naghdi equations with surface tension; Picard iterative scheme

\section{Introduction}

\subsection{General setting}

The theory of motion of free surface water waves is a vital issue that makes it a subject of many mathematical researches. The interesting part of these studies is centered on the water-waves problem of an ideal liquid, which is about describing the motion of the free surface and the evolution of the velocity field of a layer of perfect, incompressible, irrotational fluid under the influence of gravity.

Earlier work gives a useful theoretical background for this problem. This motion is described by the free surface Euler equations, which are well-posed (see [5, 10, 14, 17-19]). However, it is very hard to describe the solutions of the Euler equations; for that the Euler system is replaced by an asymptotic model.

In particular, the Green-Naghdi system presents one of them that is used in coastal oceanography $[4,6,7,9,16]$. Its range of validity depends on the physical characteristics of the flow under consideration, that is, a certain assumption is made on the dimensionless parameters, $\mu$, the shallowness parameter, and $\varepsilon$, the nonlinearity parameter, defined as

$$
\mu=\frac{h_{0}^{2}}{\lambda^{2}} \quad \text { and } \quad \varepsilon=\frac{a}{h_{0}}
$$

where $h_{0}$ is the reference depth, $\lambda$ is the wavelength of the waves and $a$ is the order of amplitude of the waves and the bottom variations. We have $\mu \ll 1$ in shallow water scaling and without a smallness assumption on $\varepsilon$, the Green-Naghdi equations are derived (see $[7,12]$ and $[2])$.

(c) The Author(s) 2018. This article is distributed under the terms of the Creative Commons Attribution 4.0 International License (http://creativecommons.org/licenses/by/4.0/), which permits unrestricted use, distribution, and reproduction in any medium, provided you give appropriate credit to the original author(s) and the source, provide a link to the Creative Commons license, and indicate if changes were made. 
The authors studied the Green-Naghdi equations in [13] and [8] with flat bottom and with non-flat bottom, respectively, using a standard Picard iterative scheme. Note that the aforementioned work is restricted to the case of null surface tension, that is, one assumed that the surface tension coefficient $\sigma$ is zero.

In the current work, we deal with the Green-Naghdi equations with non-flat bottom, and to approach the reality, we take into consideration the presence of surface tension.

In non-dimensionalized variables, we denote by $\zeta(t, x)$ and $u(t, x)$, respectively, the parametrization of the surface and the layer-mean horizontal velocity. $b(x)$ is the bottom parametrization and $b_{o}$ is the rescaled bond number, which is inversely proportional to the surface tension coefficient $\sigma$.

$b_{o}$ is given by $b_{o}=\frac{\rho g h_{0}^{2}}{\sigma}$, where $\rho$ is the density and $g$ is the gravitational force. Note that the fact that the surface tension is involved causes a higher order nonlinear model than the previous models mentioned in the case of null surface tension.

Now the Green-Naghdi equations with non-flat bottom and taking into consideration the surface tension can be written as follows:

$$
\left\{\begin{array}{l}
\partial_{t} \zeta+\nabla .(h u)=0, \\
(h+\mu h \mathcal{T}[h, \varepsilon b])\left[\partial_{t} u+\varepsilon(u . \nabla) u\right]+h\left(1-\frac{\mu}{b_{o}} \Delta\right) \nabla \zeta+\mu \varepsilon h Q[h, \varepsilon b] u=0,
\end{array}\right.
$$

where $h=1+\varepsilon(\zeta-b), \mathcal{T}[h, \varepsilon b] u=-\frac{1}{3 h} \nabla\left(h^{3} \nabla \cdot u\right)+\frac{\varepsilon}{2 h}\left[\nabla\left(h^{2} \nabla b \cdot u\right)-h^{2} \nabla b \nabla \cdot u\right]+\varepsilon^{2} \nabla b \nabla b . u$ and $Q$ is the second order differential operator defined by

$$
Q[h, \varepsilon b](u)=\frac{2}{3 h} \nabla\left(h^{3}(\nabla \cdot u)^{2}\right)+\varepsilon h(\nabla \cdot u)^{2} \nabla b+\frac{\varepsilon}{2 h} \nabla\left(h^{2} u \cdot(u \cdot \nabla) \nabla b\right)+\varepsilon^{2}(u \cdot(u \cdot \nabla) \nabla b) \nabla b .
$$

In fact, it is assumed that $b_{o}$ is not too small, so that $\frac{\mu}{b_{o}}=O(\mu)$. This assumption seems quite reasonable because, for water, to have $b_{o} \leq 1$, the reference water depth must meet $h_{0} \leq 2.7 \mathrm{~mm}$ (see [11] for more details).

Actually, the presence of surface tension in this model is represented by the term $\frac{\mu}{b_{o}} h \Delta \nabla \zeta$. Indeed the capillary term created is $\frac{\mu}{b_{o}} h \nabla \nabla \cdot\left(\frac{\nabla \zeta}{\sqrt{1+\varepsilon^{2} \mu|\nabla \zeta|^{2}}}\right)$, but taking the assumption on $b_{o}$, its asymptotic expansion becomes

$$
\frac{\mu}{b_{o}} h \nabla \nabla \cdot\left(\frac{\nabla \zeta}{\sqrt{1+\varepsilon^{2} \mu|\nabla \zeta|^{2}}}\right)=\frac{\mu}{b_{o}} h \Delta \nabla \zeta+O\left(\mu^{2} \varepsilon^{2}\right)
$$

In fact, addressing the higher derivatives, especially of third order, will make the work more difficult. The main task will be precisely how to control these terms.

\subsection{Outline of the paper}

The above model is considered a useful model in coastal oceanography since it takes into consideration dispersive effects, which are neglected in the shallow water equations. In addition the order of nonlinearity of this model is higher than the order of the Boussinesq equations.

The 1D Green-Naghdi model for flat bottoms was justified by Li [13]. After that the general case was studied by Alvarez-Samaniego and Lannes [2]. Using a Nash-Moser scheme, the well-posdness of this model was proved by Alvarez-Samaniego (see [3]). 
The results of [3] are still valid for $1 \mathrm{D}$ and 2D cases with non-flat bottoms. Note that the estimates of the linearized equations lead to loss higher derivatives, which is the main reason for using the Nash-Moser scheme in [3]. However, such losses do not occur in 1D for flat and non-flat bottoms, and so it is possible to construct a solution via a standard Picard scheme as in [13] for flat bottoms and [8] for non-flat bottoms.

The aim of this paper is to show that it is possible to construct a solution of the $1 \mathrm{D}$ Green-Naghdi equations with non-flat bottom and under the influence of surface tension (1D-GN $\sigma)$ using a Picard scheme.

\subsection{Organization of the paper}

This paper consists of three sections and an appendix. In Sect. 1, we present a general setting and some notations. In Sect. 2, the model 1D-GN $\sigma$ is presented and an energy estimate is derived. The existence, uniqueness and the conservation of energy of the model 1D-GN $\sigma$ are proved in Sect. 3. However, the proof of the existence and uniqueness of the solution of the linear Cauchy problem associated to the Green-Naghdi equations with surface tension is left to the Appendix.

\subsection{Notation}

In this section, we recall some normed vector spaces, operators and notations which will be used throughout the paper.

- $C^{k}(\mathbb{R})$ denotes the space of $k$-times continuously differentiable functions, and $C_{0}^{\infty}(\mathbb{R})$ the space of infinitely differentiable functions with compact support in $\mathbb{R}$. The space of infinitely differentiable functions which are bounded together with all their derivatives is denoted by $C_{b}^{\infty}(\mathbb{R})$.

- $L^{p}=L^{p}(\mathbb{R})$, where $0 \leq p<\infty$, is the space of all Lebesgue-measurable functions $f$ with standard norm

$$
|f|_{L^{p}}=\left(\int_{\mathbb{R}}|f(x)|^{p}\right)^{\frac{1}{p}}<\infty
$$

In case $p=2$, the norm is denoted by $|\cdot|_{L^{2}}$ or $\operatorname{simply}|\cdot|_{2}$.

The inner product of two functions $f_{1}$ and $f_{2}$ in Hilbert space $L^{2}(\mathbb{R})$ is given by

$$
\left(f_{1}, f_{2}\right)=\int_{\mathbb{R}} f_{1}(x) \overline{f_{2}(x)} d x
$$

- $L^{\infty}=L^{\infty}(\mathbb{R})$ is the space of all essentially bounded Lebesgue-measurable functions $f$ with the norm

$$
|f|_{L^{\infty}}=\operatorname{ess} \sup |f(x)|<\infty
$$

- $W^{1, \infty}=W^{1, \infty}(\mathbb{R})=\left\{f \in L^{\infty}, \partial_{x} f \in L^{\infty}\right\}$ endowed with its canonical norm.

- For all real $s, H^{s}=H^{s}(\mathbb{R})$ denotes the fractional Sobolev space of all tempered distributions $f$. Throughout this work we equip $H^{s}$ with the norm $|f|_{H^{s}}=\left|\wedge^{s} f\right|_{L^{2}}<\infty$, where $\wedge$ is the pseudo-differential operator given by $\wedge=\left(1-\partial_{x}^{2}\right)^{\frac{1}{2}}$.

- $C\left(a_{1}, a_{2}, \ldots\right)$ denotes a constant depending on the parameters $a_{1}, a_{2}, \ldots$, and whose dependence on the $a_{i}$ is always assumed to be nondecreasing. 
- $[T, f]$ is the commutator operator defined by

$$
[T, f] g=T(f g)-f T(g)
$$

where $T$ is a closed operator defined in a Banach space $X$, with $f, g$ and $f g$ belonging to the domain of $T$.

\section{Linear analysis and energy estimate}

\subsection{Presentation of the problem in 1D}

The 1D Green-Naghdi equations with non-flat bottom under the influence of surface tension (1D-GN $\sigma$ ) are given by the following:

$$
\left\{\begin{array}{l}
\partial_{t} \zeta+\partial_{x}(h u)=0 \\
(h+\mu h \mathcal{T}[h, \varepsilon b])\left[\partial_{t} u+\varepsilon u \partial_{x} u\right]+h\left(1-\frac{\mu}{b_{o}} \partial_{x}^{2}\right) \partial_{x} \zeta+\mu \varepsilon h Q[h, \varepsilon b](u)=0,
\end{array}\right.
$$

where

$$
\mathcal{T}[h, \varepsilon b] u=-\frac{1}{3 h} \partial_{x}\left(h^{3} u_{x}\right)+\frac{\varepsilon}{2 h}\left[\partial_{x}\left(h^{2} b_{x} u\right)-h^{2} b_{x} u_{x}\right]+\varepsilon^{2} b_{x}^{2} u
$$

and

$$
Q[h, \varepsilon b](u)=\frac{2}{3 h} \partial_{x}\left(h^{3} u_{x}^{2}\right)+\varepsilon h u_{x}^{2} b_{x}+\frac{\varepsilon}{2 h} \partial_{x}\left(h^{2} u^{2} b_{x x}\right)+\varepsilon^{2} u^{2} b_{x x} b_{x} .
$$

Equation (2) enables us to solve the main problem in this model which is the appearance of the third order derivatives of $u$ and $\zeta$ with respect to $x$.

\subsection{Preliminary results}

We begin this section by an important remark which arises from a physical interpretation.

Remark 1 The non-zero depth condition

$$
\exists h_{0}>0 \quad \text { such that } \quad \inf _{x \in \mathbb{R}} h \geq h_{0}, \quad \text { where } h=1+\varepsilon(\zeta-b)
$$

is valid initially, which is a necessary condition for the model 1D-GN $\sigma$ (2) to be physically valid.

Now on, we note $\mathcal{I}=h+\mu h \mathcal{T}[h, \varepsilon b]$ and $\mathcal{J}=1-\frac{\mu}{b_{o}} \partial_{x}^{2}$. Indeed, $\mathcal{I}$ and $\mathcal{J}$ will play an important role in obtaining the energy estimate and later on the local well-posedness of model (2).

Note that $\mathcal{I}: H^{2}(\mathbb{R}) \rightarrow \mathrm{七}^{2}(\mathbb{R})$ is well defined, one to one and onto (see [8]).

The following lemma gives some properties of $\mathcal{I}^{-1}$ and some essential estimates.

Lemma 1 Let $b \in C_{b}^{\infty}(\mathbb{R}), t_{0}>\frac{1}{2}$ and $\zeta \in H^{t_{0}+1}(\mathbb{R})$ be such that (3) is satisfied. Then:

(i) $\forall 0 \leq s \leq t_{0}+1$

$$
\left|\mathcal{I}^{-1} f\right|_{H^{s}}+\sqrt{\mu}\left|\partial_{x} \mathcal{I}^{-1} f\right|_{H^{s}} \leq C\left(\frac{1}{h_{0}},|h-1|_{H^{t_{0}+1}}\right)|f|_{H^{s}},
$$


(ii) $\forall 0 \leq s \leq t_{0}+1$

$$
\sqrt{\mu}\left|\mathcal{I}^{-1} \partial_{x} g\right|_{H^{s}}+\mu\left|\partial_{x} \mathcal{I}^{-1} \partial_{x} g\right|_{H^{s}} \leq C\left(\frac{1}{h_{0}},|h-1|_{H^{t_{0}+1}}\right)|g|_{H^{s}}
$$

(iii) if $s \geq t_{0}+1$ and $\zeta \in H^{s}(\mathbb{R})$ then

$$
\left\|\mathcal{I}^{-1}\right\|_{H^{s}(\mathbb{R}) \rightarrow H^{s}(\mathbb{R})}+\sqrt{\mu}\left\|\mathcal{I}^{-1} \partial_{x}\right\|_{H^{s}(\mathbb{R}) \rightarrow H^{s}(\mathbb{R})}+\mu\left\|\partial_{\mathcal{X}} \mathcal{I}^{-1} \partial_{x}\right\|_{H^{s}(\mathbb{R}) \rightarrow H^{s}(\mathbb{R})} \leq C_{s},
$$

where $C_{s}$ is a constant depending on $\frac{1}{h_{0}},|h-1|_{H^{s}}$ and independent of $\mu$ and $\varepsilon$ on $(0,1)$.

Proof The first inequality is proved in [8] and in a similar way we can prove the second and the third inequalities.

\subsection{I.V.P. model around a reference state}

In this section the model 1D-GN $\sigma$ (2) can be written in a condensed form as follows:

$$
\partial_{t} U+A^{\sigma}[U] \partial_{x} U+B(U)=0
$$

where

$$
\begin{aligned}
& U=(\zeta, u)^{t}, \quad A^{\sigma}[U]=\left(\begin{array}{cc}
\varepsilon u & h \\
\mathcal{I}^{-1} h \mathcal{J} & \varepsilon u+\mathcal{I}^{-1} Q_{1}[U]
\end{array}\right), \quad B(U)=\left(\begin{array}{c}
-\varepsilon b_{x} u \\
\mathcal{I}^{-1} q(U)
\end{array}\right), \\
& Q_{1}[U] f=\frac{2}{3} \varepsilon \mu \partial_{x}\left(h^{3} u_{x} f\right)+\varepsilon^{2} \mu h^{2} b_{x} u_{x} f+\varepsilon^{2} \mu h^{2} b_{x x} u f, \quad \text { and } \\
& q(U)=\varepsilon^{3} \mu h b_{x x} b_{x} u^{2}+\frac{1}{2} \varepsilon^{2} \mu \partial_{x}\left(h^{2} b_{x x}\right) u^{2} .
\end{aligned}
$$

In order to analyze the 1D-GN $\sigma$ model, we introduce the linearized (I.V.P.) form of the condensed equation around some reference state $\underline{U}=(\underline{\zeta}, \underline{u})^{t}$ which is given by

$$
\left\{\begin{array}{l}
\partial_{t} U+A^{\sigma}[\underline{U}] \partial_{x} U+B(\underline{U})=0 \\
U_{t=0}=U_{0} .
\end{array}\right.
$$

$X^{s}$ denotes the energy space of the linearized problem.

Definition 1 For all $s \geq 0$ and $T>0$ we denote by $X^{s}$ the vector space $H^{s+1}(\mathbb{R}) \times H^{s+1}(\mathbb{R})$ endowed with the norm

$$
\forall U=(\zeta, u)^{t} \in X^{s}, \quad|U|_{X^{s}}^{2}:=|\zeta|_{H^{s}}^{2}+|u|_{H^{s}}^{2}+\frac{\mu}{b_{o}}\left|\partial_{x} \zeta\right|_{H^{s}}^{2}+\mu\left|\partial_{x} u\right|_{H^{s}}^{2} .
$$

Meanwhile $X_{T}^{s}$ stands for $C\left(\left[0, \frac{T}{\varepsilon}\right] ; X^{s}\right)$ endowed with its canonical norm.

Definition 2 The operator $S^{\sigma}=\left(\frac{\mathcal{J}}{0} \underline{\underline{\mathcal{I}}}\right)$ is the symmetrizer of $A^{\sigma}[\underline{U}]$ where $\underline{\mathcal{J}}=\mathcal{J}$ and $\underline{\mathcal{I}}=\underline{h}+\mu \underline{h} \mathcal{T}[\underline{h}, \varepsilon b]$. The natural energy of the system I.V.P. (4) is given by

$$
E^{s}(U)^{2}=\left(\wedge^{s} U, S^{\sigma} \wedge^{s} U\right)
$$


The following lemma presents some properties for the commutator operator (for more details see [3] and [8]).

\section{Lemma 2}

(i) $\forall s>\frac{3}{2},\left|\left[\wedge^{s}, F\right] G\right|_{2} \leq c|\nabla F|_{H^{s-1}}|G|_{H^{s-1}}$ where $c$ is a constant independent of the small parameters involved.

(ii) $\forall s \geq 0, \partial_{x}\left(\left[\wedge^{s}, f\right] g\right)=\left[\wedge^{s}, f_{x}\right] g+\left[\wedge^{s}, f\right] g_{x}$ and $\partial_{x}\left[\wedge^{s}, f\right] g=\left[\wedge^{s}, \partial_{x}(f).\right] g$.

The equivalence between $E^{s}(U)$ and the norm $|\cdot|_{X^{s}}$ is given and proved in the following lemma.

Lemma 3 Let $b \in C_{b}^{\infty}(\mathbb{R}), s \geq 0$ and $\underline{\zeta} \in W^{1, \infty}(\mathbb{R})$ such that (3) is satisfied.

So we see that $E^{s}(U)$ is uniformly equivalent to the $|\cdot|_{X^{s}}$ norm with respect to $\mu$ and $\varepsilon$ on $(0,1)$, that is,

$$
C|U|_{X^{s}} \leq E^{s}(U) \leq C\left(|\underline{h}|_{L^{\infty}},\left.\underline{h}_{x}\right|_{L^{\infty}}\right)|U|_{X^{s}}
$$

where $C$ is a constant that depends only on $h_{0}$.

Proof The definition of the energy implies

$$
E^{s}(U)^{2}=\left(\wedge^{s} U, S^{\sigma} \wedge^{s} U\right)
$$

After writing $S^{\sigma}$ explicitly, we get

$$
E^{s}(U)^{2}=\left(\wedge^{s} \zeta, \underline{\mathcal{J}} \wedge^{s} \zeta\right)+\left(\wedge^{s} u, \underline{\mathcal{I}} \wedge^{s} u\right)
$$

First, we will prove that $E^{s}(U) \leq C\left(|\underline{h}|_{L^{\infty}},\left|\underline{h}_{x}\right|_{L^{\infty}}\right)|U|_{X^{s}}$. In fact, by writing $\underline{\mathcal{J}}$ and $\underline{\mathcal{I}}$ in their explicit forms and by an integration by parts, we get

$$
\begin{aligned}
\left(\wedge^{s} \zeta, \underline{\mathcal{J}} \wedge^{s} \zeta\right)= & \left(\wedge^{s} \zeta, \wedge^{s} \zeta\right)+\frac{\mu}{b_{o}}\left(\wedge^{s} \zeta_{x}, \wedge^{s} \zeta_{x}\right) \\
= & |\zeta|_{H^{s}}^{2}+\frac{\mu}{b_{o}}\left|\zeta_{x}\right|_{H^{s}}^{2}, \\
\left(\wedge^{s} u, \underline{\mathcal{I}} \wedge^{s} u\right)= & \left(\wedge^{s} u, \underline{h} \wedge^{s} u\right)+\frac{\mu}{3}\left(\wedge^{s} u_{x}, \underline{h}^{3} \wedge^{s} u_{x}\right)-\frac{\varepsilon \mu}{2}\left(\wedge^{s} u_{x}, \underline{h^{2}} b_{x} \wedge^{s} u\right) \\
& -\frac{\varepsilon \mu}{2}\left(\wedge^{s} u, \underline{h^{2}} b_{x} \wedge^{s} u_{x}\right)+\varepsilon^{2} \mu\left(\wedge^{s} u, \underline{h} b_{x}^{2} \wedge^{s} u\right) .
\end{aligned}
$$

By using the Cauchy-Schwarz inequality, one obtains the first inequality.

On the other hand, one can realize that the following equation is true:

$$
\begin{aligned}
\left(\wedge^{s} u, \underline{\mathcal{I}} \wedge^{s} u\right)= & \left(\wedge^{s} u, \underline{h} \wedge^{s} u\right) \\
& +\mu\left(\frac{\underline{h}}{\sqrt{3}} \wedge^{s} u_{x}-\frac{\sqrt{3}}{2} \varepsilon b_{x} \wedge^{s} u, \underline{h}\left(\frac{\underline{h}}{\sqrt{3}} \wedge^{s} u_{x}-\frac{\sqrt{3}}{2} \varepsilon b_{x} \wedge^{s} u\right)\right) \\
& +\frac{\varepsilon^{2} \mu}{4}\left(b_{x} \wedge^{s} u, \underline{h} b_{x} \wedge^{s} u\right) .
\end{aligned}
$$


By using the non-zero depth condition (3), one obtains

$$
\begin{aligned}
\left(\wedge^{s} u, \underline{\mathcal{I}} \wedge^{s} u\right) & \geq h_{0}\left|\wedge^{s} u\right|_{2}^{2}+\mu h_{0}\left|\frac{\underline{h}}{\sqrt{3}} \wedge^{s} u_{x}-\frac{\sqrt{3}}{2} \varepsilon b_{x} \wedge^{s} u\right|_{2}^{2}+\frac{\varepsilon^{2} \mu}{4} h_{0}\left|b_{x} \wedge^{s} u\right|_{2}^{2} \\
& \geq h_{0}\left|\wedge^{s} u\right|_{2}^{2}+\mu h_{0}\left|\frac{\underline{h}}{\sqrt{3}} \wedge^{s} u_{x}-\frac{\sqrt{3}}{2} \varepsilon b_{x} \wedge^{s} u\right|_{2}^{2}+\frac{\mu h_{0}}{3}\left|\frac{\sqrt{3}}{2} \varepsilon b_{x} \wedge^{s} u\right|_{2}^{2} \\
& \geq h_{0}\left|\wedge^{s} u\right|_{2}^{2}+2 \frac{\mu h_{0}}{6}\left[\left|\frac{\underline{h}}{\sqrt{3}} \wedge^{s} u_{x}-\frac{\sqrt{3}}{2} \varepsilon b_{x} \wedge^{s} u\right|_{2}^{2}+\left|\frac{\sqrt{3}}{2} \varepsilon b_{x} \wedge^{s} u\right|_{2}^{2}\right]
\end{aligned}
$$

Now, using the fact

$$
2\left(|A|^{2}+|B|^{2}\right) \geq(|A|+|B|)^{2} \geq|A+B|^{2}
$$

and condition (3), we get

$$
\left(\wedge^{s} u, \underline{\mathcal{I}} \wedge^{s} u\right) \geq h_{0}\left|\wedge^{s} u\right|_{2}^{2}+\frac{\mu h_{0}^{2}}{18}\left|\wedge^{s} u_{x}\right|_{2}^{2}
$$

and since we have $\left(\wedge^{s} \zeta, \underline{\mathcal{J}} \wedge^{s} \zeta\right)=|\zeta|_{H^{s}}^{2}+\frac{\mu}{b_{o}}\left|\zeta_{x}\right|_{H^{s}}^{2}$, this implies

$$
E^{S}(U) \geq|\zeta|_{H^{s}}^{2}+\frac{\mu}{b_{o}}\left|\zeta_{x}\right|_{H^{s}}^{2}+h_{0}|u|_{H^{s}}^{2}+\frac{\mu h_{0}^{2}}{18}\left|u_{x}\right|_{H^{s}}^{2}
$$

and thus one can deduce that $E^{s}(U) \geq C|U|_{X^{s}}$ where $C$ is a constant that depends only on $h_{0}$.

Remark 2 It should be noted that in this paper the bottom topography is assumed to be $C^{\infty}$ with all derivatives bounded and of the same size as the surface deformation. Indeed, such an assumption was made only for simplicity, although this assumption could be improved easily, but that is not of our interesting here.

\subsection{Energy estimates}

In this section we are going to estimate the energy $E^{s}(U)$ which is given by Proposition 1 . Later this will help us to show the well-posedness of the Green-Naghdi model 1D-GNo (2).

Proposition 1 Let $\underline{U}=(\underline{\zeta}, \underline{u})^{t} \in X_{T}^{s}$ be such that $\partial_{t} \underline{U} \in X_{T}^{s-1}$ and satisfying (3) on $\left[0, \frac{T}{\varepsilon}\right]$. If $b \in C_{b}^{\infty}(\mathbb{R}), t_{0}>\frac{1}{2}$ and $s \geq t_{0}+1$ then for all $U_{0} \in X^{s}$ there exists a unique solution $U=$ $(\zeta, u)^{t} \in X_{T}^{s}$ to (4) that satisfies the following energy estimate:

$$
E^{s}(U(t)) \leq e^{\varepsilon \lambda_{T} t} E^{s}\left(U_{0}\right)+\varepsilon \int_{0}^{t} e^{\varepsilon \lambda_{T}\left(t-t^{\prime}\right)} C\left(E^{s}(\underline{U})\left(t^{\prime}\right)\right) d t^{\prime}
$$

$\forall t \in\left[0, \frac{T}{\varepsilon}\right]$ and for some $\lambda_{T}=\lambda_{T}\left(\sup _{0 \leq t \leq \frac{T}{\varepsilon}} E^{s}(\underline{U}(t)), \sup _{0 \leq t \leq \frac{T}{\varepsilon}}\left|\partial_{t} \underline{\zeta}(t)\right|_{L^{\infty}}\right)$.

Remark 3 In fact, the constant referred to in this proposition is dependent on $\mu$, $\varepsilon$ and $\frac{1}{b_{o}}$, but we have $\mu, \varepsilon \in(0,1)$ and the assumption specified on $b_{o}$ in the introduction makes $\frac{1}{b_{o}}$ always bounded by some constant. So based on this information we can consider the constant in this proposition to be independent of $\mu, \varepsilon$ and $b_{o}$. 
Proof The existence and uniqueness of the solution to the I.V.P. (4) is achieved in the Appendix. The energy $E^{s}(U)$ satisfies

$$
\frac{1}{2} e^{\varepsilon \lambda t} \partial_{t}\left(e^{-\varepsilon \lambda t} E^{S}(U)^{2}\right)=-\frac{\varepsilon \lambda}{2} E^{s}(U)^{2}+\frac{1}{2} \partial_{t}\left(E^{S}(U)^{2}\right)
$$

and since $E^{s}(U)^{2}=\left(\wedge^{s} U, S^{\sigma} \wedge^{s} U\right)$, we get

$$
\partial_{t}\left(E^{s}(U)^{2}\right)=2\left(\wedge^{s} U_{t}, S^{\sigma} \wedge^{s} U\right)+\left(\wedge^{s} U,\left[\partial_{t}, S^{\sigma}\right] \wedge^{s} U\right) .
$$

Therefore

$$
\begin{aligned}
\frac{1}{2} e^{\varepsilon \lambda t} \partial_{t}\left(e^{-\varepsilon \lambda t} E^{s}(U)^{2}\right)= & -\frac{\varepsilon \lambda}{2} E^{s}(U)^{2}-\left(S^{\sigma} A^{\sigma}[\underline{U}] \wedge^{s} \partial_{x} U, \wedge^{s} U\right) \\
& -\left(\left[\wedge^{s}, A^{\sigma}[\underline{U}]\right] \partial_{x} U, S^{\sigma} \wedge^{s} U\right) \\
& -\left(\wedge^{s} B(\underline{U}), S^{\sigma} \wedge^{s} U\right)+\frac{1}{2}\left(\wedge^{s} U,\left[\partial_{t}, S^{\sigma}\right] \wedge^{s} U\right)
\end{aligned}
$$

Our goal is to find an upper bound on the right hand side of the last equation, and we proceed in four steps.

Step 1: Control of $A:=\left(S^{\sigma} A^{\sigma}[\underline{U}] \wedge^{s} \partial_{x} U, \wedge^{s} U\right)$.

By expanding the expression of $A$, we write

$$
\begin{aligned}
A= & \left(\varepsilon \underline{\mathcal{J}} \underline{u} \wedge^{s} \zeta_{x}, \wedge^{s} \zeta\right)+\left(\underline{\mathcal{J}} \underline{h} \wedge^{s} u_{x}, \wedge^{s} \zeta\right)+\left(\underline{h} \underline{\mathcal{J}} \wedge^{s} \zeta_{x}, \wedge^{s} u\right) \\
& +\left(\underline{\mathcal{I}} \underline{u} \wedge^{s} u_{x}, \wedge^{s} u\right)+\left(Q_{1}[\underline{U}] \wedge^{s} u_{x}, \wedge^{s} u\right) .
\end{aligned}
$$

Setting $A_{1}=\left(\varepsilon \underline{\mathcal{J}} \underline{u} \wedge^{s} \zeta_{x}, \wedge^{s} \zeta\right)$, using the explicit form of $\underline{\mathcal{J}}$ and integrating by parts, leads to

$$
A_{1}=-\frac{1}{2}\left(\varepsilon \underline{u}_{x} \wedge^{s} \zeta, \wedge^{s} \zeta\right)+\frac{\varepsilon \mu}{b_{o}}\left(\underline{u}_{x} \wedge^{s} \zeta_{x}, \wedge^{s} \zeta_{x}\right) \quad \text { and so } \quad\left|A_{1}\right| \leq \varepsilon C\left(\left|\underline{u}_{x}\right|_{L^{\infty}}\right) E^{s}(U)^{2} .
$$

Consider $A_{2}+A_{3}:=\left(\underline{\mathcal{J}} \underline{h} \wedge^{s} u_{x}, \wedge^{s} \zeta\right)+\left(\underline{h} \underline{\mathcal{J}} \wedge^{s} \zeta_{x}, \wedge^{s} u\right)$, Again, using the explicit form of $\underline{\mathcal{J}}$ and integrating by parts we get

$$
\begin{aligned}
A_{2}+A_{3}= & \left(\underline{h} \wedge^{s} u_{x}, \wedge^{s} \zeta\right)+\left(\underline{h} \wedge^{s} \zeta_{x}, \wedge^{s} u\right)-\frac{\mu}{b_{o}}\left(\underline{h}_{x x} \wedge^{s} \zeta_{x}, \wedge^{s} u\right) \\
& +\frac{\mu}{b_{o}}\left(\underline{h} \wedge^{s} u_{x x}, \wedge^{s} \zeta_{x}\right)+\frac{\mu}{b_{o}}\left(\underline{h} \wedge^{s} \zeta_{x x}, \wedge^{s} u_{x}\right)
\end{aligned}
$$

another integration by parts gives

$$
A_{2}+A_{3}=-\left(\wedge^{s} u, \underline{h}_{x} \wedge^{s} \zeta\right)-\frac{\mu}{b_{o}}\left(\wedge^{s} \zeta_{x}, \underline{h}_{x x} \wedge^{s} u\right)-\frac{\mu}{b_{o}}\left(\wedge^{s} \zeta_{x}, \underline{h}_{x} \wedge^{s} u_{x}\right)
$$

since we have $H^{s} \hookrightarrow W^{1, \infty}$, so, there is a constant $c$ so that

$$
\sqrt{\frac{\mu}{b_{o}}}\left|\underline{\zeta}_{x}\right|_{W^{1, \infty}} \leq c \sqrt{\frac{\mu}{b_{o}}}\left|\underline{\zeta}_{x}\right|_{H^{s}} \leq c E^{s}(\underline{U})
$$


Applying the Cauchy-Schwarz inequality yields

$$
\left|A_{2}+A_{3}\right| \leq \varepsilon C\left(E^{s}(\underline{U})\right) E^{s}(U)^{2}
$$

We denote $A_{4}:=\left(\underline{\mathcal{I}}\left(\underline{u} \wedge^{s} u_{x}\right), \wedge^{s} u\right)$. Combining the explicit expression of $\underline{\mathcal{I}}$ and integration by parts leads to

$$
\begin{aligned}
A_{4}:= & -\frac{\varepsilon}{2}\left((\underline{h u})_{x} \wedge^{s} u, \wedge^{s} u\right)-\frac{\varepsilon \mu}{6}\left(\left(\underline{h}^{3}\right)_{x} \underline{u} \wedge^{s} u_{x}, \wedge^{s} u_{x}\right)+\frac{\varepsilon \mu}{3}\left(\underline{h}^{3} \underline{u}_{x} \wedge^{s} u_{x}, \wedge^{s} u_{x}\right) \\
& +\frac{\varepsilon^{2} \mu}{2}\left(\underline{u} \wedge^{s} u_{x},\left(\underline{h}^{2} b_{x} \wedge^{s} u\right)_{x}\right)-\frac{\varepsilon^{2} \mu}{2}\left(\underline{h}^{2} b_{x} \underline{u} \wedge^{s} u_{x}, \wedge^{s} u_{x}\right) \\
& +\varepsilon^{3} \mu\left(\underline{h^{2}} b_{x}^{2} \underline{u} \wedge^{s} u_{x}, \wedge^{s} u\right) .
\end{aligned}
$$

Applying the Cauchy-Schwarz inequality yields

$$
\left|A_{4}\right| \leq \varepsilon C\left(|\underline{u}|_{W^{1, \infty}},|\underline{\zeta}|_{W^{1, \infty}}\right) E^{s}(U)^{2} .
$$

Now we introduce $A_{5}:=\left(Q_{1}[\underline{U}] \wedge^{s} u_{x}, \wedge^{s} u\right)$. Using the explicit expression of $Q_{1}$ and integration by parts, one obtains

$$
A_{5}:=-\frac{2}{3} \varepsilon \mu\left(\underline{h}^{3} \underline{u}_{x} \wedge^{s} u_{x}, \wedge^{s} u_{x}\right)+\varepsilon^{2} \mu\left(\underline{h}^{2} \underline{u}_{x} b_{x} \wedge^{s} u_{x}, \wedge^{s} u\right)+\varepsilon^{2} \mu\left(\underline{h}^{2} \underline{u} b_{x x} \wedge^{s} u_{x}, \wedge^{s} u\right)
$$

After applying the Cauchy-Schwarz inequality, we get

$$
\left|A_{5}\right| \leq \varepsilon C\left(|\underline{u}|_{W^{1, \infty}},|\underline{\zeta}|_{W^{1, \infty}}\right) E^{s}(U)^{2}
$$

Now, by using the fact that $H^{s}(\mathbb{R}) \subset W^{1, \infty}(\mathbb{R})$ where $s>\frac{3}{2}$, we conclude

$$
|A| \leq \varepsilon C\left(E^{s}(\underline{U})\right) E^{s}(U)^{2} .
$$

Step 2: Control of $B:=\left(\left[\wedge^{s}, A^{\sigma}[\underline{U}]\right] \partial_{x} U, S^{\sigma} \wedge^{s} U\right)$.

$$
\begin{aligned}
B= & \left(\left[\wedge^{s}, \varepsilon \underline{u}\right] \zeta_{x}, \underline{\mathcal{J}} \wedge^{s} \zeta\right)+\left(\left[\wedge^{s}, \underline{h}\right] u_{x}, \underline{\mathcal{I}} \wedge^{s} \zeta\right)+\left(\left[\wedge^{s}, \underline{\mathcal{I}}^{-1} \underline{h} \underline{\mathcal{J}}\right] \zeta_{x}, \underline{\mathcal{I}} \wedge^{s} u\right) \\
& +\left(\left[\wedge^{s}, \varepsilon \underline{u}\right] u_{x}, \underline{\mathcal{I}} \wedge^{s} u\right)+\left(\left[\wedge^{s}, \underline{\mathcal{I}}^{-1} Q_{1}[\underline{U}]\right] u_{x}, \underline{\mathcal{I}} \wedge^{s} u\right) .
\end{aligned}
$$

We can also write $B$ in another form by writing $\underline{\mathcal{J}}$ in explicit form.

$$
\begin{aligned}
B= & \left(\left[\wedge^{s}, \varepsilon \underline{u}\right] \zeta_{x}, \wedge^{s} \zeta\right)+\left(\left[\wedge^{s}, \underline{h}\right] u_{x}, \wedge^{s} \zeta\right)+\left(\left[\wedge^{s}, \varepsilon \underline{u}\right] u_{x}, \underline{\mathcal{I}} \wedge^{s} u\right) \\
& +\left(\left[\wedge^{s}, \underline{\mathcal{I}}^{-1} \underline{h}\right] \zeta_{x}, \underline{\mathcal{I}} \wedge^{s} u\right)+\left(\left[\wedge^{s}, \underline{\mathcal{I}}^{-1} Q_{1}[\underline{U}]\right] u_{x}, \underline{\mathcal{I}} \wedge^{s} u\right) \\
& -\frac{\mu}{b_{o}}\left(\left[\wedge^{s}, \varepsilon \underline{u}\right] \zeta_{x}, \wedge^{s} \zeta_{x x}\right)-\frac{\mu}{b_{o}}\left(\left[\wedge^{s}, \underline{h}\right] u_{x}, \wedge^{s} \zeta_{x x}\right) \\
& -\frac{\mu}{b_{o}}\left(\left[\wedge^{s}, \underline{\mathcal{I}}^{-1} \underline{h} \partial_{x}^{2}\right] \zeta_{x}, \underline{\mathcal{I}} \wedge^{s} u\right) .
\end{aligned}
$$

Consider $B_{1}+B_{2}+B_{3}:=\left(\left[\wedge^{s}, \varepsilon \underline{u}\right] \zeta_{x}, \wedge^{s} \zeta\right)+\left(\left[\wedge^{s}, \underline{h}\right] u_{x}, \wedge^{s} \zeta\right)+\left(\left[\wedge^{s}, \varepsilon \underline{u}\right] u_{x}, \underline{\mathcal{I}} \wedge^{s} u\right)$. 
Now, by writing the explicit form of $\underline{\mathcal{I}}$, integration by parts, Lemma 2 and the CauchySchwarz inequality, we get

$$
\left|B_{1}+B_{2}+B_{3}\right| \leq \varepsilon C\left(E^{s}(\underline{U})\right) E^{s}(U)^{2} .
$$

Consider $B_{4}:=\left(\left[\wedge^{s}, \underline{\mathcal{I}}^{-1} \underline{h}\right] \zeta_{x}, \underline{\mathcal{I}} \wedge^{s} u\right)$.

Note that $\underline{\mathcal{I}}\left[\wedge^{s}, \underline{\mathcal{I}}^{-1} \underline{h}\right] \zeta_{x}=-\left[\wedge^{s}, \underline{\mathcal{I}}\right] \underline{\mathcal{I}}^{-1} \underline{h} \zeta_{x}+\left[\wedge^{s}, \underline{h}\right] \zeta_{x}$.

By using the explicit form of $\underline{\mathcal{I}}$ and Lemma 2 , one can check that

$$
\begin{aligned}
\underline{\mathcal{I}}\left[\wedge^{s}, \underline{\mathcal{I}}^{-1} \underline{h}\right] \zeta_{x}= & -\left[\wedge^{s}, \underline{h}\right] \underline{\mathcal{I}}^{-1} \underline{h} \zeta_{x}+\frac{\mu}{3} \partial_{x}\left(\left[\wedge^{s}, \underline{h}^{3}\right] \partial_{x}\left(\underline{\mathcal{I}}^{-1} \underline{h} \zeta_{x}\right)\right)-\frac{\varepsilon \mu}{2} \partial_{x}\left(\left[\wedge^{s}, \underline{h}^{2} b_{x}\right] \underline{\mathcal{I}}^{-1} \underline{h} \zeta_{x}\right) \\
& +\frac{\varepsilon \mu}{2}\left[\wedge^{s}, \underline{h}^{2} b_{x}\right] \partial_{x} \underline{\mathcal{I}}^{-1} \underline{h} \zeta_{x}-\varepsilon^{2} \mu\left[\wedge^{s}, \underline{h} b_{x}^{2}\right] \underline{\mathcal{I}}^{-1} \underline{h} \zeta_{x}+\left[\wedge^{s}, \underline{h}\right] \zeta_{x} .
\end{aligned}
$$

After using integration by parts, one obtains

$$
\begin{aligned}
B_{4}= & : B_{41}+B_{42}+B_{43}+B_{44}+B_{45}+B_{46} \\
= & -\left(\left[\wedge^{s}, \underline{h}\right] \underline{\mathcal{I}}^{-1} \underline{h} \zeta_{x}, \wedge^{s} u\right)-\frac{\mu}{3}\left(\left[\wedge^{s}, \underline{h}^{3}\right] \partial_{x}\left(\underline{\mathcal{I}}^{-1} \underline{h} \zeta_{x}\right), \wedge^{s} u_{x}\right) \\
& +\frac{\varepsilon \mu}{2}\left(\left[\wedge^{s}, \underline{h}^{2} b_{x}\right] \underline{\mathcal{I}}^{-1} \underline{h} \zeta_{x}, \wedge^{s} u_{x}\right)+\frac{\varepsilon \mu}{2}\left(\left[\wedge^{s}, \underline{h}^{2} b_{x}\right] \partial_{x}\left(\underline{\mathcal{I}}^{-1} \underline{h} \zeta_{x}\right), \wedge^{s} u\right) \\
& -\varepsilon^{2} \mu\left(\left[\wedge^{s}, \underline{h} b_{x}^{2}\right] \underline{\mathcal{I}}^{-1} \underline{h} \zeta_{x}, \wedge^{s} u\right)+\left(\left[\wedge^{s}, \underline{h}\right] \zeta_{x}, \wedge^{s} u\right) .
\end{aligned}
$$

Note that, since we have $\underline{U}=(\zeta, \underline{u})^{t} \in X_{T}^{s}$ and $U=(\zeta, u)^{t} \in X_{T}^{s}$, one can check that all terms in $B_{4}$ created by $\left[\wedge^{s}, \underline{\mathcal{I}}\right] \underline{\mathcal{I}}^{-1} \underline{h} \zeta_{x}$ make sense.

Note that $H^{s} \hookrightarrow H^{s-1}$ and we have the following estimate:

$$
\left|\partial_{x}\left(\underline{\mathcal{I}}^{-1} \underline{h} \zeta_{x}\right)\right|_{H^{s-1}} \leq c\left|\underline{\mathcal{I}}^{-1} \underline{h} \zeta_{x}\right|_{H^{s}} \leq c\left\|\underline{\mathcal{I}}^{-1}\right\|_{H^{s}(R) \rightarrow H^{s}(R)}\left|\underline{h} \zeta_{x}\right|_{H^{s}}
$$

where $c$ is a constant. Now, taking into consideration these estimates:

$$
\left|\underline{h} \zeta_{x}\right|_{H^{s-1}} \leq C\left(E^{s}(\underline{U})\right) E^{s}(U) \quad \text { and } \quad\left|\underline{h}^{2} b_{x}\right|_{H^{s}}+\left|\underline{h} b_{x}^{2}\right|_{H^{s}} \leq C\left(E^{s}(\underline{U})\right)
$$

by using Lemma 1, Lemma 2 and the Cauchy-Schwarz inequality, one can obtain

$$
\left|B_{4}\right| \leq \varepsilon C\left(E^{s}(\underline{U})\right) E^{s}(U)^{2} .
$$

Consider $B_{5}:=\left(\left[\wedge^{s}, \underline{\mathcal{I}}^{-1} Q_{1}[\underline{U}]\right] u_{x}, \underline{\mathcal{I}} \wedge^{s} u\right)$.

Remark that $\underline{\mathcal{I}}\left[\wedge^{s}, \underline{\mathcal{I}}^{-1} Q_{1}[\underline{U}]\right] u_{x}=-\left[\wedge^{s}, \underline{\mathcal{I}}\right] \underline{\mathcal{I}}^{-1} Q_{1}[\underline{U}] u_{x}+\left[\wedge^{s}, Q_{1}[\underline{U}]\right] u_{x}$; one can use the explicit expression of $\mathcal{I}$ and Lemma 2 to get

$$
\begin{aligned}
\mathcal{I}\left[\wedge^{s}, \underline{\mathcal{I}}^{-1} Q_{1}[\underline{U}]\right] u_{x}= & -\left[\wedge^{s}, \underline{h}\right] \underline{I}^{-1} Q_{1}[\underline{U}] u_{x}+\frac{\mu}{3} \partial_{x}\left(\left[\wedge^{s}, \underline{h}^{3}\right] \partial_{x}\left(\underline{\mathcal{I}}^{-1} Q_{1}[\underline{U}] u_{x}\right)\right) \\
& -\frac{\varepsilon \mu}{2} \partial_{x}\left(\left[\wedge^{s}, \underline{h^{2}} b_{x}\right] \underline{\mathcal{I}}^{-1} Q_{1}[\underline{U}] u_{x}\right) \\
& +\frac{\varepsilon \mu}{2}\left[\wedge^{s}, \underline{h}^{2} b_{x}\right] \partial_{x}\left(\underline{\mathcal{I}}^{-1} Q_{1}[\underline{U}] u_{x}\right) \\
& -\varepsilon^{2} \mu\left[\wedge^{s}, \underline{h}^{2} b_{x}\right] \underline{\mathcal{I}}^{-1} Q_{1}[\underline{U}] u_{x}+\left[\wedge^{s}, Q_{1}[\underline{U}]\right] u_{x} .
\end{aligned}
$$


By applying integration by parts, one obtains

$$
\begin{aligned}
B_{5}= & -\left(\left[\wedge^{s}, \underline{h}\right] \underline{\mathcal{I}}^{-1} Q_{1}[\underline{U}] u_{x}, \wedge^{s} u\right)-\frac{\mu}{3}\left(\left[\wedge^{s}, \underline{h}^{3}\right] \partial_{x}\left(\underline{\mathcal{I}}^{-1} Q_{1}[\underline{U}] u_{x}\right), \wedge^{s} u_{x}\right) \\
& +\frac{\varepsilon \mu}{2}\left(\left[\wedge^{s}, \underline{h}^{2} b_{x}\right] \underline{\mathcal{I}}^{-1} Q_{1}[\underline{U}] u_{x}, \wedge^{s} u_{x}\right)+\frac{\varepsilon \mu}{2}\left(\left[\wedge^{s}, \underline{h}^{2} b_{x}\right] \partial_{x}\left(\underline{\mathcal{I}}^{-1} Q_{1}[\underline{U}] u_{x}\right), \wedge^{s} u\right) \\
& -\varepsilon^{2} \mu\left(\left[\wedge^{s}, \underline{h}^{2} b_{x}\right] \underline{\mathcal{I}^{-1}} Q_{1}[\underline{U}] u_{x}, \wedge^{s} u\right)+\left(\left[\wedge^{s}, Q_{1}[\underline{U}]\right] u_{x}, \wedge^{s} u\right) ;
\end{aligned}
$$

remember that the explicit form of $Q_{1}[\underline{U}]$ is

$$
Q_{1}[\underline{U}] w=\frac{2}{3} \varepsilon \mu \partial_{x}\left(\underline{h}^{3} \underline{u}_{x} w\right)+\varepsilon^{2} \mu \underline{h}^{2} b_{x} \underline{u}_{x} w+\varepsilon^{2} \mu \underline{h}^{2} b_{x x} \underline{u} w .
$$

In fact, since we have $\underline{U}=(\underline{\zeta}, \underline{u})^{t} \in X_{T}^{s}$ and $U=(\zeta, u)^{t} \in X_{T}^{s}$, one can check that all terms in $B_{5}$ created by $\left[\wedge^{s}, \underline{\mathcal{I}}\right] \underline{\mathcal{I}}^{-1} Q_{1}[\underline{U}] u_{x}$ make sense.

Now, by writing $Q_{1}[\underline{U}]$ in explicit form and noting that the following estimate:

$$
\left|\partial_{x}\left(\underline{\mathcal{I}}^{-1} \partial_{x}\left(\underline{h}^{3} \underline{u}_{x} u_{x}\right)\right)\right|_{H^{s-1}} \leq c\left|\underline{\mathcal{I}}^{-1} \partial_{x}\left(\underline{h}^{3} \underline{u}_{x} u_{x}\right)\right|_{H^{s}} \leq c\left\|\underline{\mathcal{I}}^{-1} \partial_{x}\right\|_{H^{s}(R) \rightarrow H^{s}(R)}\left|\underline{h}^{3} \underline{u}_{x} u_{x}\right|_{H^{s}},
$$

where $c$ is a constant. So, the control of these terms becomes clear and evident. In fact using Lemma 1, Lemma 2, $H^{s} \hookrightarrow H^{s-1}$ and the Cauchy-Schwarz inequality, one can deduce

$$
\left|B_{5}\right| \leq \varepsilon C\left(E^{s}(\underline{U})\right) E^{s}(U)^{2} .
$$

Set $B_{6}=-\frac{\mu}{b_{o}}\left(\left[\wedge^{s}, \varepsilon \underline{u}\right] \partial_{x} \zeta, \wedge^{s} \partial_{x}^{2} \zeta\right)$ and $B_{7}=-\frac{\mu}{b_{o}}\left(\left[\wedge^{s}, \underline{h}\right] u_{x}, \wedge^{s} \zeta_{x x}\right)$.

So, by integration by parts and using the commutator properties of the estimation, we get

$$
\begin{aligned}
& B_{6}=\frac{\mu}{b_{o}}\left(\left[\wedge^{s}, \varepsilon \underline{u}_{x}\right] \zeta_{x}, \wedge^{s} \zeta_{x}\right)+\frac{\mu}{b_{o}}\left(\left[\wedge^{s}, \varepsilon \underline{u}\right] \zeta_{x x}, \wedge^{s} \zeta_{x}\right), \\
& B_{7}=\frac{\mu}{b_{o}}\left(\left[\wedge^{s}, \underline{h}_{x}\right] u_{x}, \wedge^{s} \zeta_{x}\right)+\frac{\mu}{b_{o}}\left(\left[\wedge^{s}, \underline{h}\right] u_{x x}, \wedge^{s} \zeta_{x}\right) .
\end{aligned}
$$

By applying the commutator estimate, the Cauchy-Schwarz inequality and the fact that $\left|\zeta_{x x}\right|_{H^{s-1}} \leq c\left|\zeta_{x}\right|_{H^{s}}$ and $\left|u_{x x}\right|_{H^{s-1}} \leq c\left|u_{x}\right|_{H^{s}}$ (where $c$ is a constant), one obtains

$$
\left|B_{6}+B_{7}\right| \leq \varepsilon C\left(E^{s}(\underline{U})\right) E^{s}(U)^{2} .
$$

However, to bound $B_{8}:=\frac{\mu}{b_{o}}\left(\left[\wedge^{s}, \underline{\mathcal{I}}^{-1} \underline{h} \partial_{x}^{2}\right] \zeta_{x}, \underline{\mathcal{I}} \wedge^{s} u\right)$, we remark that $\underline{\mathcal{I}}\left[\wedge^{s}, \underline{\mathcal{I}}^{-1} \underline{h} \partial_{x}^{2}\right] \zeta_{x}=$ $-\left[\wedge^{s}, \underline{\mathcal{I}}\right] \underline{\mathcal{I}}^{-1} \underline{h} \zeta_{x x x}+\left[\wedge^{s}, \underline{h}\right] \zeta_{x x x}$ and use Lemma 2.

Thus, we obtain

$$
\begin{aligned}
B_{8}= & -\frac{\mu}{b_{o}}\left(\left[\wedge^{s}, \underline{h}\right] \zeta_{x x}, \wedge^{s} u_{x}\right)-\frac{\mu}{b_{o}}\left(\left[\wedge^{s}, \underline{h}_{x}\right] \zeta_{x x}, \wedge^{s} u\right) \\
& -\frac{\mu}{b_{o}}\left(\left[\wedge^{s}, \underline{\mathcal{I}}\right] \underline{\mathcal{I}}^{-1} \partial_{x}\left(\underline{h} \zeta_{x x}\right), \wedge^{s} u\right)+\frac{\mu}{b_{o}}\left(\left[\wedge^{s}, \underline{\mathcal{I}}\right] \underline{\mathcal{I}}^{-1} \underline{h}_{x} \zeta_{x x}, \wedge^{s} u\right) .
\end{aligned}
$$

Consider $B_{81}+B_{82}=-\frac{\mu}{b_{o}}\left(\left[\wedge^{s}, \underline{h}\right] \zeta_{x x}, \wedge^{s} u_{x}\right)-\frac{\mu}{b_{o}}\left(\left[\wedge^{s}, \underline{h}_{x}\right] \zeta_{x x}, \wedge^{s} u\right)$. 
Lemma 2 and the Cauchy-Schwarz inequality imply that

$$
\left|B_{81}+B_{82}\right| \leq \varepsilon C\left(E^{s}(\underline{U})\right) E^{s}(U)^{2} .
$$

We introduce $B_{83}:=-\frac{\mu}{b_{0}}\left(\left[\wedge^{s}, \underline{\mathcal{I}}\right] \underline{\mathcal{I}}^{-1} \partial_{x}\left(\underline{h} \zeta_{x x}\right), \wedge^{s} u\right)$ and $B_{84}:=\frac{\mu}{b_{0}}\left(\left[\wedge^{s}, \underline{\mathcal{I}}\right] \underline{\mathcal{I}}^{-1} \underline{h}_{x} \zeta_{x x}, \wedge^{s} u\right)$.

In order to control the term $B_{83}$, we write $\mathcal{I}$ in explicit form; using Lemma 2 and by integration by parts, we get

$$
\begin{aligned}
B_{83}=: & B_{831}+B_{832}+B_{833}+B_{834}+B_{835} \\
= & -\frac{\mu}{b_{o}}\left(\left[\wedge^{s}, \underline{h}\right] \underline{\mathcal{I}}^{-1} \partial_{x}\left(\underline{h} \zeta_{x x}\right), \wedge^{s} u\right)-\frac{\mu^{2}}{3 b_{o}}\left(\left[\wedge^{s}, \underline{h}^{3}\right] \partial_{x} \underline{\mathcal{I}}^{-1} \partial_{x}\left(\underline{h} \zeta_{x x}\right), \wedge^{s} u_{x}\right) \\
& +\frac{\varepsilon \mu^{2}}{2 b_{o}}\left(\left[\wedge^{s}, \underline{h^{2}} b_{x}\right] \underline{\mathcal{I}}^{-1} \partial_{x}\left(\underline{h} \zeta_{x x}\right), \wedge^{s} u_{x}\right)+\frac{\varepsilon \mu^{2}}{2 b_{o}}\left(\left[\wedge^{s}, \underline{h}^{2} b_{x}\right] \partial_{x} \underline{\mathcal{I}^{-1}} \partial_{x}\left(\underline{h} \zeta_{x x}\right), \wedge^{s} u\right) \\
& -\frac{\varepsilon^{2} \mu^{2}}{b_{o}}\left(\left[\wedge^{s}, \underline{h} b_{x}^{2}\right] \underline{\mathcal{I}}^{-1} \partial_{x}\left(\underline{h} \zeta_{x x}\right), \wedge^{s} u\right) ;
\end{aligned}
$$

of course, one can check that all the terms in $B_{83}$ created by $\frac{\mu}{b_{o}}\left[\wedge^{s}, \underline{\mathcal{I}}\right] \underline{\mathcal{I}}^{-1} \partial_{x}\left(\underline{h} \zeta_{x x}\right)$ make sense, and that is done because we have $\underline{U}=(\underline{\zeta}, \underline{u})^{t} \in X_{T}^{s}$ and $U=(\zeta, u)^{t} \in X_{T}^{s}$.

Remark that the following estimates:

$$
\begin{aligned}
& \left|\underline{\mathcal{I}}^{-1} \partial_{x}\left(\underline{h} \zeta_{x x}\right)\right|_{H^{s-1}} \leq c\left\|\underline{\mathcal{I}}^{-1} \partial_{x}\right\|_{H^{s-1} \rightarrow H^{s-1}}\left|\underline{h} \zeta_{x x}\right|_{H^{s-1}}, \\
& \left|\partial_{x}\left(\underline{\mathcal{I}}^{-1} \partial_{x}\left(\underline{h} \zeta_{x x}\right)\right)\right|_{H^{s-1}} \leq c\left\|\partial_{x} \underline{\mathcal{I}^{-1}} \partial_{x}\right\|_{H^{s-1} \rightarrow H^{s-1}}\left|\underline{h} \zeta_{x x}\right|_{H^{s-1}},
\end{aligned}
$$

hold, where $c$ is a constant. Actually, by using Lemma 1, Lemma 2 and the CauchySchwarz inequality, one can obtain

$$
\begin{aligned}
& \left|B_{831}\right| \leq C\left(\frac{1}{h_{0}},|\underline{h}-1|_{H^{s}}\right)\left|\underline{h}_{x}\right|_{H^{s-1}} \sqrt{\frac{\mu}{b_{o}}}\left|\underline{h} \zeta_{x x}\right|_{H^{s-1}}|u|_{H^{s}}, \\
& \left|B_{832}\right| \leq C\left(\frac{1}{h_{0}},|\underline{h}-1|_{H^{s}}\right)\left|\left(\underline{h}^{3}\right)_{x}\right|_{H^{s-1}} \sqrt{\frac{\mu}{b_{o}}}\left|\underline{h} \zeta_{x x}\right|_{H^{s-1}} \sqrt{\mu}\left|u_{x}\right|_{H^{s}}, \\
& \left|B_{833}\right| \leq\left.\varepsilon C\left(\frac{1}{h_{0}},|\underline{h}-1|_{H^{s}}\right)\left|\left(\underline{h}^{2} b_{x}\right)_{x}\right|_{H^{s-1}} \sqrt{\frac{\mu}{b_{o}}} \underline{\mid h} \zeta_{x x}\right|_{H^{s-1}} \sqrt{\mu}\left|u_{x}\right|_{H^{s}}, \\
& \left|B_{834}+B_{835}\right| \leq \varepsilon C\left(\frac{1}{h_{0}}, \underline{h}-\left.1\right|_{H^{s}}\right)\left(\left|\left(\underline{h^{2}} b_{x}\right)_{x}\right|_{H^{s-1}}+\left|\left(\underline{h} b_{x}^{2}\right)_{x}\right|_{H^{s-1}}\right) \sqrt{\frac{\mu}{b_{o}}}\left|\underline{h} \zeta_{x x}\right|_{H^{s-1}}|u|_{H^{s}},
\end{aligned}
$$

and after remarking that $H^{s} \hookrightarrow H^{s-1}$, one concludes that $\left|B_{83}\right| \leq \varepsilon C\left(E^{s}(\underline{U})\right) E^{s}(U)^{2}$.

Similarly, by writing $\underline{\mathcal{I}}$ in explicit form, using Lemma 2 and integration by parts, we get

$$
\begin{aligned}
B_{84}= & : B_{841}+B_{842}+B_{843}+B_{844}+B_{845}+B_{846}+B_{847} \\
= & \frac{\mu}{b_{o}}\left(\left[\wedge^{s}, \underline{h}\right] \underline{\mathcal{I}}^{-1} \underline{h}_{x} \zeta_{x x}, \wedge^{s} u\right) \\
& +\frac{\mu^{2}}{3 b_{o}}\left(\left[\wedge^{s}, \underline{h}^{3}\right] \partial_{x}\left(\underline{\mathcal{I}}^{-1} \partial_{x}\left(\underline{h}_{x} \zeta_{x}\right)\right), \wedge^{s} u_{x}\right)-\frac{\mu^{2}}{3 b_{o}}\left(\left[\wedge^{s}, \underline{h}^{3}\right] \partial_{x}\left(\underline{\mathcal{I}}^{-1} \partial_{x}\left(\underline{h}_{x}\right) \zeta_{x}\right), \wedge^{s} u_{x}\right)
\end{aligned}
$$




$$
\begin{aligned}
& -\frac{\varepsilon \mu^{2}}{2 b_{o}}\left(\left[\wedge^{s}, \underline{h}^{2} b_{x}\right] \underline{I}^{-1} \underline{h}_{x} \zeta_{x x}, \wedge^{s} u_{x}\right)+\frac{\varepsilon \mu^{2}}{2 b_{o}}\left(\left[\wedge^{s}, \underline{h}^{2} b_{x}\right] \underline{I}^{-1} \underline{h}_{x} \zeta_{x x}, \wedge^{s} u_{x}\right) \\
& +\frac{\varepsilon \mu^{2}}{2 b_{o}}\left(\left[\wedge^{s},\left(\underline{h}^{2} b_{x}\right)_{x}\right] \underline{\mathcal{I}}^{-1} \underline{h}_{x} \zeta_{x x}, \wedge^{s} u\right)-\frac{\varepsilon^{2} \mu^{2}}{b_{o}}\left(\left[\wedge^{s}, \underline{h} b_{x}^{2}\right] \underline{I}^{-1} \underline{h}_{x} \zeta_{x x}, \wedge^{s} u\right) ;
\end{aligned}
$$

also, all the terms in $B_{84}$ created by $\frac{\mu}{b_{0}}\left[\wedge^{s}, \underline{\mathcal{I}}\right] \underline{\mathcal{I}}^{-1} \underline{h}_{x} \zeta_{x x}$ make sense, and that is done because we have $\underline{U}=(\underline{\zeta}, \underline{u})^{t} \in X_{T}^{s}$ and $U=(\zeta, u)^{t} \in X_{T}^{s}$.

In particular, remark that the following estimates:

$$
\begin{aligned}
& \left|\underline{\mathcal{I}}^{-1} \underline{h}_{x} \zeta_{x x}\right|_{H^{s-1}} \leq\left\|\underline{\mathcal{I}}^{-1}\right\|_{H^{s-1} \rightarrow H^{s-1}}\left|\underline{h}_{x} \zeta_{x x}\right|_{H^{s-1}}, \\
& \left|\partial_{x}\left(\underline{\mathcal{I}}^{-1}\left(\partial_{x}\left(\underline{h}_{x} \zeta_{x}\right)\right)\right)\right|_{H^{s-1}} \leq\left\|\partial_{x} \underline{\mathcal{I}}^{-1} \partial_{x}\right\|_{H^{s-1} \rightarrow H^{s-1}}\left|\underline{h}_{x} \zeta_{x}\right|_{H^{s-1}},
\end{aligned}
$$

hold, and by using Lemma 1, Lemma 2 and the Cauchy-Schwarz inequality, one obtains

$$
\begin{aligned}
&\left|B_{841}\right| \leq C\left(\frac{1}{h_{0}},|\underline{h}-1|_{H^{s}}\right)\left|\underline{h}_{x}\right|_{H^{s-1}} \sqrt{\frac{\mu}{b_{o}}}\left|\underline{h}_{x} \zeta_{x x}\right|_{H^{s-1}}|u|_{H^{s}}, \\
&\left|B_{842}+B_{843}\right| \leq C\left(\frac{1}{h_{0}},|\underline{h}-1|_{H^{s}}\right)\left|\left(\underline{h^{3}}\right)_{x}\right|_{H^{s-1}}\left|\underline{h}_{x} \zeta_{x}\right|_{H^{s-1}} \sqrt{\mu}\left|u_{x}\right|_{H^{s}} \\
&\left|B_{844}+B_{845}\right| \leq \varepsilon C\left(\frac{1}{h_{0}},|\underline{h}-1|_{H^{s}}\right)\left|\left(\underline{h^{2}} b_{x}\right)_{x}\right|_{H^{s-1}} \sqrt{\frac{\mu}{b_{o}}}\left|\underline{h}_{x} \zeta_{x x}\right|_{H^{s-1}} \sqrt{\mu}\left|u_{x}\right|_{H^{s}} \\
&\left|B_{846}+B_{847}\right| \leq \varepsilon C\left(\frac{1}{h_{0}},|\underline{h}-1|_{H^{s}}\right)\left(\sqrt{\frac{\mu}{b_{o}}}\left|\left(\underline{h}^{2} b_{x}\right)_{x x}\right|_{H^{s-1}}+\left|\left(\underline{h}^{2} b_{x}\right)_{x}\right|_{H^{s-1}}\right) \\
& \times \sqrt{\frac{\mu}{b_{o}}}\left|\underline{h}_{x} \zeta_{x x}\right|_{H^{s-1}}|u|_{H^{s}} .
\end{aligned}
$$

Since $H^{s} \hookrightarrow H^{s-1}$, one obtains $\left|B_{84}\right| \leq \varepsilon C\left(E^{s}(\underline{U})\right) E^{s}(U)^{2}$. Thus, one concludes

$$
|B| \leq \varepsilon C\left(E^{s}(\underline{U})\right) E^{s}(U)^{2} .
$$

Step 3: Control of $C:=\left(\wedge^{s} B(\underline{U}), S^{\sigma} \wedge^{s} U\right)$.

Here by writing $B(\underline{U})$ and $S^{\sigma}$ in their explicit forms, we get

$$
C=-\varepsilon\left(\wedge^{s}\left(b_{x} \underline{u}\right), \underline{\mathcal{J}} \wedge^{s} \zeta\right)-\left(\left[\wedge^{s}, \underline{\mathcal{I}}\right] \underline{\mathcal{I}}^{-1} q(\underline{U}), \wedge^{s} u\right)+\left(\wedge^{s} q(\underline{U}), \wedge^{s} u\right)
$$

consider $C_{1}=-\varepsilon\left(\wedge^{s}\left(b_{x} \underline{u}\right), \underline{\mathcal{J}} \wedge^{s} \zeta\right)$, write $\underline{\mathcal{J}}$ in explicit form and integrate by parts, and we get

$$
C_{1}=: C_{11}+C_{12}=-\varepsilon\left(\wedge^{s}\left(b_{x} \underline{u}\right), \wedge^{s} \zeta\right)-\frac{\varepsilon \mu}{b_{o}}\left(\wedge^{s}\left(b_{x} \underline{u}\right)_{x}, \wedge^{s} \zeta_{x}\right)
$$

and the Cauchy-Schwarz inequality implies

$$
\left|C_{11}\right| \leq c \varepsilon\left|b_{x} \underline{u}\right|_{H^{s}}|\zeta|_{H^{s}} \quad \text { and } \quad\left|C_{12}\right| \leq c \varepsilon \sqrt{\mu}\left|\left(b_{x} \underline{u}\right)_{x}\right|_{H^{s}} \sqrt{\frac{\mu}{b_{o}}}\left|\zeta_{x}\right|_{H^{s}}
$$

where $c$ is a constant. Thus, one concludes $\left|C_{1}\right| \leq \varepsilon C\left(E^{s}(\underline{U})\right) E^{s}(U)$. 
Now in order to control $C_{2}=-\left(\left[\wedge^{s}, \underline{\mathcal{I}}\right] \underline{\mathcal{I}}^{-1} q(\underline{U}), \wedge^{s} u\right)+\left(\wedge^{s} q(\underline{U}), \wedge^{s} u\right)$ writing $\underline{\mathcal{I}}$ in explicit form, using Lemma 2 and integration by parts, one obtains

$$
\begin{aligned}
C_{2}= & -\left(\left[\wedge^{s}, \underline{h}\right] \underline{\mathcal{I}}^{-1} q(\underline{U}), \wedge^{s} u\right)-\frac{\mu}{3}\left(\left[\wedge^{s}, \underline{h}^{3}\right] \partial_{x}\left(\underline{\mathcal{I}}^{-1} q(\underline{U})\right), \wedge^{s} u_{x}\right) \\
& +\frac{\varepsilon \mu}{2}\left(\left[\wedge^{s}, \underline{h}^{2} b_{x}\right] \underline{\mathcal{I}}^{-1} q(\underline{U}), \wedge^{s} u_{x}\right) \\
& \left.+\frac{\varepsilon \mu}{2}\left(\left[\wedge^{s}, \underline{h}^{2} b_{x}\right] \partial_{x} \underline{\mathcal{I}^{-1}} q \underline{\underline{U}}\right), \wedge^{s} u\right)-\varepsilon^{2} \mu\left(\left[\wedge^{s}, \underline{h} b_{x}^{2}\right] \underline{\mathcal{I}}^{-1} q(\underline{U}), \wedge^{s} u\right) \\
& +\left(\wedge^{s} q(\underline{U}), \wedge^{s} u\right) .
\end{aligned}
$$

Now, by writing $q(\underline{U})$ in explicit form, $q(\underline{U})=\varepsilon^{3} \mu \underline{h} b_{x x} b_{x} \underline{u^{2}}+\frac{1}{2} \varepsilon^{2} \mu \partial_{x}\left(\underline{h}^{2} b_{x x}\right) \underline{u^{2}}$ and using Lemma 1, Lemma 2 and the Cauchy-Schwarz inequality, one can check easily that these terms are under control and one deduces that $\left|C_{2}\right| \leq \varepsilon C\left(E^{s}(\underline{U})\right) E^{s}(U)$. Thus, one concludes

$$
|C| \leq \varepsilon C\left(E^{s}(\underline{U})\right) E^{s}(U) .
$$

Step 4: Control of $D:=\frac{1}{2}\left(\wedge^{s} U,\left[\partial_{t}, S^{\sigma}\right] \wedge^{s} U\right)$.

By writing $S^{\sigma}$ in explicit form, we obtain

$$
D=\frac{1}{2}\left(\wedge^{s} \zeta,\left[\partial_{t}, \underline{\mathcal{J}}\right] \wedge^{s} \zeta\right)+\frac{1}{2}\left(\wedge^{s} u,\left[\partial_{t}, \underline{\mathcal{I}}\right] \wedge^{s} u\right)
$$

Obviously, $D_{1}=\frac{1}{2}\left(\wedge^{s} \zeta,\left[\partial_{t}, \underline{\mathcal{J}}\right] \wedge^{s} \zeta\right)$ is equal to zero due to the form of $\underline{\mathcal{J}}$. On the other hand, we have $D_{2}=\frac{1}{2}\left(\wedge^{s} u,\left[\partial_{t}, \underline{\mathcal{I}}\right] \wedge^{s} u\right)$.

After writing $\underline{\mathcal{I}}$ in explicit form, using Lemma 2 and integration by parts, we obtain

$$
\begin{aligned}
D_{2}= & \frac{1}{2}\left(\wedge^{s} u, \underline{h}_{t} \wedge^{s} u\right)+\frac{\mu}{6}\left(\wedge^{s} u_{x},\left(\underline{h}^{3}\right)_{t} \wedge^{s} u_{x}\right)-\frac{\varepsilon \mu}{4}\left(\wedge^{s} u_{x},\left(\underline{h}^{2}\right)_{t} b_{x} \wedge^{s} u\right) \\
& -\frac{\varepsilon \mu}{4}\left(\wedge^{s} u,\left(\underline{h}^{2}\right)_{t} b_{x} \wedge^{s} u_{x}\right)+\frac{\varepsilon^{2} \mu}{2}\left(\wedge^{s} u, \underline{h}_{t} b_{x}^{2} \wedge^{s} u\right) .
\end{aligned}
$$

Therefore, by using the Cauchy-Schwarz inequality, one concludes

$$
|D| \leq \varepsilon C\left(E^{S}(\underline{U}), \mid \partial_{t} \underline{\zeta} L_{L^{\infty}}\right) E^{S}(U)^{2} .
$$

After gathering all information provided by the above estimates, we get

$$
e^{\varepsilon \lambda t} \partial_{t}\left(e^{-\varepsilon \lambda t} E^{s}(U)^{2}\right) \leq \varepsilon\left(C\left(E^{s}(\underline{U}),\left|\partial_{t} \underline{\zeta}\right|_{L^{\infty}}\right)-\lambda\right) E^{s}(U)^{2}+\varepsilon C\left(E^{s}(\underline{U}) E^{s}(U) .\right.
$$

We take $\lambda=\lambda_{T}$ large enough for some $T$ (depending on $\left.\sup _{0 \leq t \leq \frac{T}{\varepsilon}} C\left(E^{s}(\underline{U}),\left|\partial_{t} \underline{\zeta}(t)\right|_{L^{\infty}}\right)\right)$ to have the first term of the right hand side of the previous inequality negative $\forall t \in\left[0, \frac{T}{\varepsilon}\right]$.

So, we conclude that

$$
e^{\varepsilon \lambda t} \partial_{t}\left(e^{-\varepsilon \lambda t} E^{S}(U)^{2}\right) \leq \varepsilon C\left(E^{S}(\underline{U}) E^{S}(U)\right. \text {. }
$$

Finally, integrating this differential inequality yields $\forall t \in\left[0, \frac{T}{\varepsilon}\right]$

$$
E^{s}(U(t)) \leq e^{\varepsilon \lambda T^{t}} E^{s}\left(U_{0}\right)+\varepsilon \int_{0}^{t} e^{\varepsilon \lambda_{T}\left(t-t^{\prime}\right)} C\left(E^{s}(\underline{U})\left(t^{\prime}\right)\right) d t^{\prime} .
$$




\section{Well-posedness of 1D-GN $\sigma$}

The main results are presented in this section, that is, showing the well-posedness of the 1D-GN $\sigma$ model in Theorem 1 and obtaining the energy conservation in Proposition 2.

Theorem 1 Let $U_{0}=\left(\zeta_{0}, u_{0}\right)^{t} \in X^{s}$ satisfy (3), $b \in C_{b}^{\infty}(\mathbb{R}), t_{0}>\frac{1}{2}$ and $s \geq t_{0}+1$. Then the model $1 D-G N \sigma$ (2) admits a unique solution $U=(\zeta, u)^{t} \in X_{T_{\max }}^{s}$, with the initial condition $\left(\zeta_{0}, u_{0}\right)^{t}$, and preserving (3) for any $t \in\left[0, \frac{T_{\max }}{\varepsilon}\right.$ ), for some $T_{\max }>0$ (maximal instant) uniformly bounded from below with respect to $\varepsilon, \mu, \frac{1}{b_{o}} \in(0,1)$.

In the case $T_{\max }<\infty$ and as $t$ tends to $\frac{T_{\max }}{\varepsilon}$, we have

$$
|U(t, \cdot)|_{X^{s}} \rightarrow \infty \quad \text { or } \quad \inf _{\mathbb{R}} h(t, \cdot)=\inf _{\mathbb{R}} 1+\varepsilon(\zeta(t, \cdot)-b(\cdot)) \rightarrow 0 .
$$

Remark 4 The previous result shows the well-posedness only by using a standard Picard iterative and thus there is no loss of regularity of the solution with respect to the initial condition. Also this theorem has extra importance as it does not impose any smallness assumption on the parameters $\mu$ and $\varepsilon$, and this is due to the uniform boundedness of $T_{\max }$. In fact if some smallness assumption is made on $\varepsilon$ for instance, then the existence time becomes larger.

Proof Consider a sequence $\left(U^{n}=\left(\zeta^{n}, u^{n}\right)\right)_{n \geq 0}$ defined by

$$
U^{0}=U_{0} \quad \text { and } \quad \forall n \in \mathbb{N}, \quad\left\{\begin{array}{l}
\partial_{t} U^{n+1}+A^{\sigma}\left[U^{n}\right] \partial_{x} U^{n+1}+B\left(U^{n}\right)=0 \\
U_{l_{t=0}^{n+1}}^{n}=U_{0}
\end{array}\right.
$$

Let us prove recursively that (5) has a unique solution $U^{n+1} \in C\left([0, \infty) ; X^{S}\right)$ satisfying (3) and $\partial_{t} U^{n+1} \in X_{T}^{s-1}$ for all times.

For $n=0$, take $U^{0}=U_{0} \in X_{T}^{s}$, so $\partial_{t} U^{0}=0 \in X_{T}^{s-1}$ and satisfying (3), so $U^{0}$ is the unique solution for (5) for $n=0$.

Now suppose that is true till order $n-1$, which implies that the system

$$
\left\{\begin{array}{l}
\partial_{t} U^{n}+A^{\sigma}\left[U^{n-1}\right] \partial_{x} U^{n}+B\left(U^{n-1}\right)=0 \\
U_{\mid t=0}^{n}=U_{0}
\end{array}\right.
$$

has a unique solution $U^{n} \in X_{T}^{s}$ satisfying (3) and $\partial_{t} U^{n} \in X_{T}^{s-1}$.

Then by using Proposition 1 the system

$$
\left\{\begin{array}{l}
\partial_{t} U^{n+1}+A^{\sigma}\left[U^{n}\right] \partial_{x} U^{n+1}+B\left(U^{n}\right)=0 \\
U_{l_{t=0}^{n+1}}^{n+1}=U_{0}
\end{array}\right.
$$

has a unique solution $U^{n+1} \in X_{T}^{s}$. Now let us prove that it satisfies (3).

Let $M>0$ be such that $E^{s}\left(U_{0}\right) \leq \frac{M}{2}$. By using the estimate of Proposition 1, we get

$$
E^{s}\left(U^{n+1}(t)\right) \leq e^{\varepsilon \lambda_{T} t} E^{s}\left(U_{0}\right)+\varepsilon \int_{0}^{t} e^{\varepsilon \lambda_{T}\left(t-t^{\prime}\right)} C\left(E^{s}\left(U^{n}\right)\left(t^{\prime}\right)\right) d t^{\prime}
$$


Now suppose that $\sup _{t \in\left[0, \frac{T}{\varepsilon}\right]} E^{s}\left(U^{n}(t)\right) \leq M$, so one can deduce using the above estimate that there is $T>0$ such that

$$
\sup _{t \in\left[0, \frac{T}{\varepsilon}\right]} E^{s}\left(U^{n+1}(t)\right) \leq M
$$

also Lemma 3, yields

$$
\sup _{t \in\left[0, \frac{T}{\varepsilon}\right]}\left|U^{n+1}(t)\right|_{X^{s}} \leq C M
$$

and we know that $\partial_{t} \zeta^{n+1}=-h^{n} u_{x}^{n+1}-\varepsilon \zeta_{x}^{n+1} u^{n}+\varepsilon b_{x} u^{n}$, so one obtains

$$
\left|\partial_{t} h^{n+1}\right|_{L^{\infty}}=\varepsilon\left|\partial_{t} \zeta^{n+1}\right|_{L^{\infty}} \leq \varepsilon C M
$$

Using the latter inequality and the fact that $h^{n+1}=h_{t=0}^{n+1}+\varepsilon \int_{0}^{t} \partial_{t} \zeta^{n+1}$ with $h_{t=0}^{n+1} \geq h_{0}$ implies $U^{n+1}$ satisfies condition (3) for some $T$ small enough. Indeed, small to replace $h_{0}$ by $\frac{h_{0}}{2}$.

Now, it is small enough to prove that $\partial_{t} U^{n+1} \in X_{T}^{s-1}$. We have

$$
\partial_{t} U^{n+1}=-A^{\sigma}\left[U^{n}\right] \partial_{x} U^{n+1}-B\left(U^{n}\right)
$$

by writing $A^{\sigma}\left[U^{n}\right]$ and $B\left(U^{n}\right)$ in explicit form, one obtains

$$
\partial_{t} U^{n+1}=\left(\begin{array}{c}
-\varepsilon u^{n} \zeta_{x}^{n+1}-h^{n} u_{x}^{n+1}+\varepsilon b_{x} u^{n} \\
-\mathcal{I}_{n}^{-1} h^{n} \mathcal{J} \zeta_{x}^{n+1}-\varepsilon u^{n} u_{x}^{n+1}-\mathcal{I}_{n}^{-1} Q_{1}\left[U^{n}\right] u_{x}^{n+1}-\mathcal{I}_{n}^{-1} q\left(U^{n}\right)
\end{array}\right)
$$

$X^{s-1}$ is the vector space $H^{s}(\mathbb{R}) \times H^{s}(\mathbb{R})$ endowed with the norm

$$
\forall U=(\zeta, u)^{t} \in X^{s-1}, \quad|U|_{X^{s-1}}^{2}:=|\zeta|_{H^{s-1}}^{2}+|u|_{H^{s-1}}^{2}+\frac{\mu}{b_{o}}\left|\partial_{x} \zeta\right|_{H^{s-1}}^{2}+\mu\left|\partial_{x} u\right|_{H^{s-1}}^{2},
$$

while $X_{T}^{s-1}$ stands for $C\left(\left[0, \frac{T}{\varepsilon}\right] ; X^{s-1}\right)$ endowed with its canonical norm.

First we can remark that $\partial_{t} \zeta^{n+1}=-\varepsilon u^{n} \zeta_{x}^{n+1}-h^{n} u_{x}^{n+1}+\varepsilon b_{x} u^{n} \in H^{s}(\mathbb{R})$, and that is easily done, where $U^{n} \in X_{T}^{s}$ and $U^{n+1} \in X_{T}^{s}$.

Now, let us prove that

$$
\partial_{t} u^{n+1}=-\mathcal{I}_{n}^{-1} h^{n} \mathcal{J} \zeta_{x}^{n+1}-\varepsilon u^{n} u_{x}^{n+1}-\mathcal{I}_{n}^{-1} Q_{1}\left[U^{n}\right] u_{x}^{n+1}-\mathcal{I}_{n}^{-1} q\left(U^{n}\right) \in H^{s}(\mathbb{R})
$$

In fact, let us show the controlling on $-\mathcal{I}_{n}^{-1} h^{n} \mathcal{J} \zeta_{x}^{n+1}$, since it involves a higher derivative mainly of third order. We have $-\mathcal{I}_{n}^{-1} h^{n} \mathcal{J} \zeta_{x}^{n+1}=-\mathcal{I}_{n}^{-1} h^{n} \zeta_{x}^{n+1}+\frac{\mu}{b_{o}} \mathcal{I}_{n}^{-1}\left(h^{n} \zeta_{x x}^{n+1}\right)-\frac{\mu}{b_{o}} \times$ $\mathcal{I}_{n}^{-1} \partial_{x}\left(h_{x}^{n} \zeta_{x x}^{n+1}\right)$; by using the explicit form of $\mathcal{I}_{n}$ and Lemma 1 one can obtain $-\mathcal{I}_{n}^{-1} h^{n} \times$ $\mathcal{J} \zeta_{x}^{n+1} \in H^{s}$.

However, by referring to the explicit forms of $Q_{1}\left[U^{n}\right]$ and $q\left(U^{n}\right)$, one can easily check that

$$
-\varepsilon u^{n} u_{x}^{n+1}-\mathcal{I}_{n}^{-1} Q_{1}\left[U^{n}\right] u_{x}^{n+1}-\mathcal{I}_{n}^{-1} q\left(U^{n}\right) \in H^{s}(\mathbb{R})
$$


and so we conclude that

$$
\partial_{t} u^{n+1}=-\mathcal{I}_{n}^{-1} h^{n} \mathcal{J} \zeta_{x}^{n+1}-\varepsilon u^{n} u_{x}^{n+1}-\mathcal{I}_{n}^{-1} Q_{1}\left[U^{n}\right] u_{x}^{n+1}-\mathcal{I}_{n}^{-1} q\left(U^{n}\right) \in H^{s}(\mathbb{R})
$$

since we have $\left|\partial_{t} U^{n+1}\right|_{X^{s-1}}^{2} \leq c\left(\left|\partial_{t} \zeta^{n+1}\right|_{H^{s}}^{2}+\left|\partial_{t} u^{n+1}\right|_{H^{s}}^{2}\right)$ for some positive constant $c$. Thus, one concludes $\partial_{t} U^{n+1} \in X_{T}^{s-1}$.

Finally, by using Proposition 1, we conclude that for every natural number the iterative scheme (5) has a unique solution $U^{n+1}$ preserving (3) and satisfying

$$
E^{S}\left(U^{n+1}(t)\right) \leq e^{\varepsilon \lambda_{T} t} E^{S}\left(U_{0}\right)+\varepsilon \int_{0}^{t} e^{\varepsilon \lambda_{T}\left(t-t^{\prime}\right)} C\left(E^{S}\left(U^{n}\right)\left(t^{\prime}\right)\right) d t^{\prime}
$$

and $t \in\left[0, \frac{T}{\varepsilon}\right]$ and $\lambda_{T}$ depending only on $\sup _{t \in\left[0, \frac{T}{\varepsilon}\right]} E^{s}\left(U^{n}\right)$.

Now one can deduce that there exists $T_{\max }=T\left(E^{s}\left(U_{0}\right)\right)>0$, such that the system (2) has a unique solution $U \in X_{T_{\max }}^{s}$, preserving (3) for any $t \in\left[0, \frac{T_{\max }}{\varepsilon}\right]$, which is the limit of the iterative scheme (5) (see [1]).

Remark 5 It should be noted that the result is consistent with what has been established in the absence of surface tension, which was studied in [8]. In fact our approach was in 1D, while the second dimension is still an open work and is not discussed in this paper.

Proposition 2 Let $U=(\zeta, u)^{t} \in X_{T_{\max }}^{s}$ be the solution of (2), then $U$ satisfies the following equation for energy conservation: $\partial_{t}\left(|\zeta|_{2}^{2}+\frac{\mu}{b_{o}}\left|\zeta_{x}\right|_{2}^{2}+(h u, u)+\mu(h \mathcal{T} u, u)\right)=0$ where $\mathcal{T}=$ $\mathcal{T}[h, \varepsilon b]$.

Proof We multiply the first equation of (2) by $\zeta$ and the second equation by $u$. By integrating on $\mathbb{R}$ and adding both equations, we get

$$
\begin{aligned}
& \frac{1}{2} \partial_{t}|\zeta|_{2}^{2}+\left(\partial_{x}(h u), \zeta\right)+\left(\partial_{t} u, h u\right)+\mu\left(h \mathcal{T} \partial_{t} u, u\right)+\varepsilon\left(u, \partial_{x} u, h u\right) \\
& \quad+\left(\partial_{x} \zeta, h u\right)-\frac{\mu}{b_{o}}\left(\partial_{x}^{3} \zeta, h u\right)+\mu \varepsilon(\mathcal{P} u, u)=0
\end{aligned}
$$

where

$$
\begin{aligned}
\mathcal{P} u= & -\frac{1}{3} \partial_{x} h^{3}\left(u \partial_{x}^{2} u-\left(\partial_{x} u\right)^{2}\right)+\frac{\varepsilon}{2}\left[\partial_{x}\left(h^{2} u \partial_{x}\left(u b_{x}\right)\right)-h^{2} \partial_{x} b\left(u \partial_{x}^{2} u-\left(\partial_{x} u\right)^{2}\right)\right] \\
& +\varepsilon^{2} h \partial_{x} b\left(u \partial_{x}\left(u \partial_{x}\left(u b_{x}\right)\right)\right) .
\end{aligned}
$$

Now, by using the equality $h=1+\varepsilon(\zeta-b)$ and the first equation of (2), one obtains $-\frac{1}{2}\left(\partial_{t} h, u^{2}\right)+\varepsilon\left(u \partial_{x} u, h u\right)=-\frac{\varepsilon}{2}\left(\partial_{x}(h u), u^{2}\right)+\varepsilon\left(u \partial_{x} u, h u\right)=0$.

Also, by using the first equation of (2) and integration by parts, we get $-\frac{\mu}{b_{o}}\left(\partial_{x}^{3} \zeta, h u\right)=$ $\frac{\mu}{2 b_{o}} \partial_{t}\left(\left|\partial_{x} \zeta\right|_{2}^{2}\right)$. Thus, one can conclude

$$
\frac{1}{2} \partial_{t}|\zeta|_{2}^{2}+\frac{\mu}{2 b_{o}} \partial_{t}\left|\zeta_{x}\right|_{2}^{2}+\frac{1}{2} \partial_{t}(h u, u)+\mu\left(h \mathcal{T} \partial_{x} u, u\right)+\mu \varepsilon(\mathcal{P} u, u)=0
$$


Note that $\mu\left(h \mathcal{T} \partial_{t} u, u\right)=\partial_{t}(h \mathcal{T} u, u)-\frac{\mu}{2}\left(\partial_{t} h,\left(\mathcal{T}_{1} u\right)^{2}+\left(\mathcal{T}_{2} u\right)^{2}\right)-\mu\left(h \partial_{t} \mathcal{T}_{1} u, \mathcal{T}_{1} u\right)$ where $\mathcal{T}_{1} u=$ $\frac{h}{\sqrt{3}} \partial_{x} u-\frac{\varepsilon \sqrt{3}}{2} \partial_{x} b u$ and $\mathcal{T}_{2} u=\frac{\varepsilon}{2} \partial_{x} b u$ are defined in [8]. So, we have

$$
\begin{aligned}
\partial_{t} & \left(|\zeta|_{2}^{2}+\left|\zeta_{x}\right|_{2}^{2}+(h u, u)+\mu(h \mathcal{T} u, u)\right) \\
\quad= & \frac{\mu}{2}\left(\partial_{t} h,\left(\mathcal{T}_{1} u\right)^{2}+\left(\mathcal{T}_{2} u\right)^{2}\right)+\mu\left(h \partial_{t} \mathcal{T}_{1} u, \mathcal{T}_{1} u\right)-\mu \varepsilon(\mathcal{P} u, u)
\end{aligned}
$$

and since $\frac{\mu}{2}\left(\partial_{t} h,\left(\mathcal{T}_{1} u\right)^{2}+\left(\mathcal{T}_{2} u\right)^{2}\right)+\mu\left(h \partial_{t} \mathcal{T}_{1} u, \mathcal{T}_{1} u\right)-\mu \varepsilon(\mathcal{P} u, u)=0$ (see [8]), the proof is done.

\section{Conclusion}

It was necessary to take care of surface tension and a non-flat bottom when proving the existence and uniqueness of the Green-Naghdi equations in this work. The reason is that the neglecting of these external circumstances is not a simulation of nature and reality. Indeed, in some environments such conditions exist and may affect the accuracy of the solutions, so that this study will give more accuracy. In the current work, the existence and uniqueness of the Green-Naghdi equations with non-flat bottom and under the influence of surface tension is proved at a large instant of time. The Picard iterative scheme is used to prove this result where the solution is regular relative to the initial condition. The main task was the appearance of the higher derivative, mainly of third order, in a nonlinear equation. Now, and after proving the existence of that solution, the main question that is raised is if anyone can express the explicit form of this solution knowing that the nonlinear equation in this model involves a higher derivative of third order. This will be a point of study for future work.

\section{Appendix: Linearized equation study}

Let us prove the existence, uniqueness and regularity of the solution of the following system:

$$
\left\{\begin{array}{l}
\partial_{t} U+A^{\sigma}[\underline{U}] \partial_{x} U=f \\
U_{\left.\right|_{t=0}}=U_{0}
\end{array}\right.
$$

where $\underline{U}=(\underline{\zeta}, \underline{u})^{t} \in X_{T}^{s}$ is such that $\partial_{t} \underline{U} \in X_{T}^{s-1}$ and satisfies (3) on [0, $\left.\frac{T}{\varepsilon}\right]$.

We use here the same strategy used in [8], that is, we seek to obtain a solution to (6) as a limit of solutions $U_{\delta}$ to

$$
\left\{\begin{array}{l}
\partial_{t} U_{\delta}+J^{\delta} A^{\sigma}[\underline{U}] J^{\delta} \partial_{x} U_{\delta}=f \\
U_{\delta_{\mid t=0}}=U_{0}
\end{array}\right.
$$

where $J^{\delta}=\varphi(\delta|D|)$ where $\delta>0$ and $\varphi \in C_{o}^{\infty}(\mathbb{R})$ such that $\varphi(r)=1$ for $|r| \leq 1$.

The following lemma contains some properties of $J^{\delta}$ (for more details see [15] and [8]).

Lemma 4 Let $f \in C^{1}(\mathbb{R}) \cap L^{\infty}(\mathbb{R})$ and $v \in L^{2}(\mathbb{R})$ and $\forall s, s^{\prime} \in R$.

(i) $J^{\delta}: H^{s}(\mathbb{R}) \rightarrow H^{s^{\prime}}(\mathbb{R})$ is a bounded linear and self adjoint operator, and it commutes with $\wedge^{s}$. 
(ii) $\left|\left[f, J^{\delta}\right] v\right|_{H^{1}} \leq C|f|_{C^{1}}|v|_{2}$ for some constant $C$ independent of $\delta$.

Now $A_{\delta}^{\sigma}=J^{\delta} A^{\sigma}[\underline{U}] J^{\delta}$ is a bounded linear operator on each $X^{s}$ and $F_{\delta}^{\sigma}=A_{\delta}^{\sigma} \partial_{x}+B(\underline{U}) \in$ $C^{1}\left(X^{s}\right)$, so by Cauchy-Lipschitz the O.D.E. (7) has a unique solution $U_{\delta} \in C\left(\left[0, \frac{T}{\varepsilon}\right], X^{s}\right)$.

Here the task is to obtain an estimate of $U_{\delta}$ independent of $\delta \in(0,1)$ and to show that $U_{\delta} \rightarrow U$ as $\delta \rightarrow 0$ where $\mathrm{U}$ is the solution to (6). First, remark that

$$
\frac{1}{2} \partial_{t}\left(E^{s}\left(U_{\delta}\right)^{2}\right)=\left(\wedge^{s} \partial_{t} U_{\delta}, S^{\sigma} \wedge^{s} U_{\delta}\right)+\frac{1}{2}\left(\wedge^{s} U_{\delta},\left[\partial_{t}, S^{\sigma}\right] \wedge^{s} U_{\delta}\right) .
$$

By using Eq. (7) to find the expression of $\partial_{t} U_{\delta}$, we get

$$
\begin{aligned}
\frac{1}{2} \partial_{t}\left(E^{s}(U)^{2}\right)= & -\left(\wedge^{s} J^{\delta} A^{\sigma}[\underline{U}] J^{\delta} \partial_{x} J^{\delta} U_{\delta}, S^{\sigma} \wedge^{s} U_{\delta}\right)+\left(\wedge^{s} f, S^{\sigma} \wedge^{s} U_{\delta}\right) \\
& +\frac{1}{2}\left(\wedge^{s} U_{\delta},\left[\partial_{t}, S^{\sigma}\right] \wedge^{s} U_{\delta}\right) .
\end{aligned}
$$

By using the definition of the commutator estimate, we get

$$
\begin{aligned}
\frac{1}{2} \partial_{t}\left(E^{s}\left(U_{\delta}\right)^{2}\right)= & -\left(S^{\sigma} A^{\sigma}[\underline{U}] \wedge^{s} \partial_{x} J^{\delta} U_{\delta}, \wedge^{s} J^{\delta} U_{\delta}\right)-\left(\left[\wedge^{s}, A^{\sigma}[\underline{U}]\right] \partial_{x} \delta^{\delta} U_{\delta}, S^{\sigma} \wedge^{s} J^{\delta} U\right) \\
& -\left(\wedge^{s} f, S^{\sigma} \wedge^{s} U_{\delta}\right)+\frac{1}{2}\left(\wedge^{s} U,\left[\partial_{t}, S^{\sigma}\right] \wedge^{s} U_{\delta}\right) \\
& +\left(\left[S^{\sigma}, J^{\delta}\right] \wedge^{s} U_{\delta}, A^{\sigma}[\underline{U}] \wedge^{s} \partial_{x} J^{\delta} U_{\delta}\right) \\
& +\left(\left[S^{\sigma}, J^{\delta}\right] \wedge^{s} U_{\delta},\left[\wedge^{s}, A^{\sigma}[\underline{U}]\right] \partial_{x} \delta^{\delta} U_{\delta}\right) .
\end{aligned}
$$

Now, by using the same way used in order to prove Proposition 1 , that is, to control each term of the above equation, and by using Lemma 4 , one can obtain

$$
\partial_{t}\left(E^{s}\left(U_{\delta}\right)^{2}\right) \leq C E^{s}\left(U_{\delta}\right)^{2}+C E^{s}(f)^{2} .
$$

Thus Gronwall's inequality yields $E^{s}\left(U_{\delta}\right)^{2} \leq C(t)\left[\left(E^{s}\left(U_{0}\right)^{2}\right)+\sup _{t \in[0, t]} E^{s}(f)^{2}\right]$ independent of $\delta \in(0,1)$.

By using this energy estimate, we can conclude the existence and uniqueness of the solution $U \in C\left([0,1], X^{s}\right)$ to $(6)$ (see $\left.[15]\right)$.

Acknowledgements

Not applicable.

Funding

This paper is partially supported by Lebanese University.

Availability of data and materials

Not applicable.

Competing interests

The authors declare that they have no competing interests.

Authors' contributions

$\mathrm{MH}$ proved the well posedness, TEA derived the new model and SI suggested a symmetrizer for the system. All authors read and approved the final manuscript.

\section{Author details}

${ }^{1}$ Department of Mathematics and Computer Science, Faculty of Science, Beirut Arab University, Beirut, Lebanon.

${ }^{2}$ Departement of Mathematics, Faculty of Science 1, Lebanese University, Beirut, Lebanon. 


\section{Publisher's Note}

Springer Nature remains neutral with regard to jurisdictional claims in published maps and institutional affiliations.

Received: 31 January 2018 Accepted: 31 August 2018 Published online: 12 September 2018

\section{References}

1. Alinhac, S., Gérard, P.: Opérateurs pseudo-différentiels et théorème de Nash-Moser. Savoirs Actuels. InterEditions, Paris; Editions du Centre National de la Recherche Scientifique (CNRS), Meudon (1991)

2. Alvarez-Samaniego, B., Lannes, D.: Large time existence for 3D water-waves and asymptotics. Invent. Math. 171 485-541 (2008)

3. Alvarez-Samaniego, B., Lannes, D.: A Nash-Moser theorem for singular evolution equations. Application to the Serre and Green-Naghdi equations. Indiana Univ. Math. J. 57, 97-131 (2008)

4. Basenkova, S.V., Morozov, N.N., Pogutse, O.P.: Dispersive effects in two-dimensional hydrodynamics. Dokl. Akad. Nauk SSSR (1985)

5. Craig, W.: An existence theory for water waves and the Boussinesq and the Korteweg-de Vries scaling limits. Commun. Partial Differ. Equ. 10, 787-1003 (1985)

6. Green, A.E., Laws, N., Naghdi, P.M.: On the theory of water waves. Proc. R. Soc. Lond. Ser. A 338, 43-55 (1974)

7. Green, A.E., Naghdi, P.M.: A derivation of equations for wave propagation in water of variable depth. J. Fluid Mech. 78, 237-246 (1976)

8. Israwi, S.: Large time existence for 1D Green-Naghdi equations. Nonlinear Anal. 74, 83-93 (2011)

9. Kim, J.W., Bai, K.J., Ertekin, R.C., Webster, W.C.: A strongly-nonlinear model for water waves in water of variable depth: the irrotational Green-Naghdi model. J. Offshore Mech. Arct. Eng. 125(1), 25-32 (2003)

10. Lannes, D.: Well-posedness of the water waves equations. J. Am. Math. Soc. 18, 605-654 (2005)

11. Lannes, D.: The Water Waves Problem. Am. Math. Soc., Providence (2013)

12. Lannes, D., Bonneton, P.: Derivation of asymptotic two-dimensional time-dependent equations for surface water wave propagation. Phys. Fluids 21, 016601 (2009)

13. Li, Y.A.: A shallow-water approximation to the full water wave problem. Commun. Pure Appl. Math. 59, 1225-1285 (2006)

14. Nalimov, V.I.: The Cauchy-Poisson problem. Dinamika Splošn. Sredy Vyp. 18 Dinamika Zidkost. so Svobod. Granicami 254, 104-210 (1974) (Russian)

15. Taylor, M.E.: Partial Differential Equations II. Applied Mathematical Sciences, vol. 116. Springer, New York (2011)

16. Wei, G., Kirby, J.T., Grilli, S.T., Subramanya, R.: A fully nonlinear Boussinesq model for surface waves. Part I. Highly nonlinear unsteady waves. J. Fluid Mech. 294, 71-92 (1995)

17. Wu, S.: Well-posedness in Sobolev spaces of the full water wave problem in 2-D. Invent. Math. 130(1), 39-72 (1997)

18. Wu, S.: Well-posedness in Sobolev spaces of the full water wave problem in 3-D. J. Am. Math. Soc. 12(2), 445-495 (1999)

19. Yosihara, H.: Gravity waves on the free surface of an incompressible perfect fluid of finite depth. Publ. Res. Inst. Math. Sci. 18(1), 49-96 (1982)

\section{Submit your manuscript to a SpringerOpen ${ }^{\circ}$ journal and benefit from:}

- Convenient online submission

- Rigorous peer review

- Open access: articles freely available online

- High visibility within the field

- Retaining the copyright to your article

Submit your next manuscript at $\gg$ springeropen.com 